\title{
Evaluation of patient's satisfaction towards medical care provided at Civil Hospital, Palanpur: Assessed by direct approach exit interview method
}

\author{
Dhiraj Trivedi ${ }^{*}$, Pratik Trivedi $^{2}$ \\ ${ }^{1}$ Professor, ${ }^{2} \mathrm{Jr}$ Store Manager, ${ }^{1}$ Dept. of Biochemistry, Zydus Medical college and Hospital, Dahod, Gujarat, India \\ *Corresponding Author: Dhiraj Trivedi \\ Email: dhiraj99trivedi@gmail.com
}

\begin{abstract}
Satisfaction means achieving one's expectation regarding service or product. Pateint's satisfaction means fulfilling their expectations during medical care. Total 211 patient's response towards medical care at civil hospital was assessed by direct approach exit interview method. Present study finds more than $60 \%$ responders were satisfied regards to reception and admission procedure. More than $70 \%$ were not happy with quality of food, cleanliness and rules of the hospital. Above 52\% respoonders disapproved behaviour of ward and sanitary attendents but $70 \%$ plus responders appreciated other health care workers in their soft skill. Over $60 \%$ responders expressed satisfaction in medical treatment and preventive education for disease they suffer. Satisfaction level was low for discharge protocol and time.

Overall impression of the present study display satisfactory response towards services and quality of health care provided by the Civil Hospital. It identify a few shortfalls, which need to be attended for improving quality of health care services and to maintain acceptable standard of the hospital. Though survey evaluates quality of health care services of Civil hospital from patients' point of view but, it facilitate identification of problem and may provide feedback to management for quality improvement towards maintaining standard of Hospital Services.
\end{abstract}

Keywords: Patient's satisfaction, Medical care, Civil Hospital.

\section{Introduction}

Satisfaction means achieving one's expectation regarding service or product. When Satisfaction regards to patient, it means fulfilling the expectation while regaining his health in clean environment under caring hands at reasonable cost. Though it looks simple but various factors are in between patient's expectation and satisfaction. ${ }^{1}$ At time patient visit a busy, popular healthcare provider with some expectations and returns discontented because he feels that health care person has not listened and attended him by giving sufficient time, though he has received good medical treatment. Now and then patient may land up in highly reputed, busy hospital which charges high fees for its clean environment and caring staff but patient feel unhappy because hospital staff and environment stands low. On the other hand, it happens that patient reaches to low cost hospital like Govt hospital or benevolent health centre and feels satisfied. It may be because expectations from such centre are minimal. ${ }^{2}$ Thus satisfaction depends on expectations and can vary from person to person or from community or group. ${ }^{3}$

Corporate and private hospitals run on high fees and modern infrastructure having patient's satisfaction as primary motto. As high expectation of service is a positive indicator of reputation in community. It is important in getting popularity, attracting customers and increasing business. On the other hand Government hospitals are running on low cost and people have a belief that, they are less concerned about consumer's expectation and not apprehensive about satisfaction of their customer. Health Care providing team at government hospital feels that, due to free to almost negligible charges paid by patient; hospital finds constrain in providing quality treatment to match patient's satisfaction. Also it is true that as patients pay less, their expectations from medical care at such centre is minimal. The present day competition and consumer law have changed medical care; even government hospitals are not spared. Health care providers have to change their approach towards patients comfort and quality care. ${ }^{17}$ Hence patients are benefited even at government set ups to meet their expectations.

In present study we evaluated patient's satisfaction level at civil hospital, Palanpur by taking direct approach exit interview method to collect response.

\section{Materials and Methods \\ Place of study}

Civil hospital, Palanpur is situated at Banaskantha District of North Gujarat. It is old hospital established during the era of Nawab Tale Mohmad. Hospital receives patients from villages of North Gujarat and touching areas of Rajasthan. Hospital has 10 departments which provide Outpatient (OPD) consultations and inpatient services to patient visiting the hospital. On an average 70 inpatients and 400 OPD patients are utilizing hospital services per day.

\section{Study design and Sampling}

Present study is cross sectional and descriptive type, which follows random convenient sampling method. Study population includes indoor patients above 18 years of age of either gender. Patients below 18 years were considered minor and excluded from the study. All indoor patient of infective disease wards were also excluded from the study population.

By using statistical formula $\left[\mathrm{n}=\mathrm{Z}^{2} \mathrm{pq} / \mathrm{d}^{2}\right]$, minimum sample size estimated ${ }^{4}$ was 211 . Also this number was about 2 to $3 \%$ of total indoor patients admitted during six months of our study. 
Pretested structured questionnaires ${ }^{5,6}$ were prepared keeping 3 points in focus viz. 1. Environment: Reception, restraints and Cleanliness in hospital. 2. Soft skill: Approach and behaviour of health care staff. 3. Quality: Medical treatment quality and education on patient's illness while discharge.

Ethical clearance was obtained from hospital authority. Informed consent was acquired from each participant who volunteered for the study. Pretested questionnaire were given to each patient a day prior to their discharge from the hospital. Inputs were obtained on the day of their discharge. Specially trained staff nurse was allotted this work of data collection.

The participants were asked to respond by tick mark on any one of the four options (Excellent, Good, Average, or Poor). Average and Poor response together were considered 'dissatisfied' while Excellent and Good option were considered as 'satisfied' category. ${ }^{5}$

Data obtained from 211 participants by direct approach on exit interview from questionnaire were analysed by MedCal online statistical software.

\section{Result}

In six months (i.e. October 2017 to March 2018) of our study period total 10,030 indoor patients were admitted to Civil Hospital, Palanpur. Among these patients 212 randomly selected patients volunteered to participate in our study giving written consent. This makes $2.11 \%$ of indoor patients becoming participants. Out of 212 subjects 50.94\% were male and $49.06 \%$ were female ( $\mathrm{M}$ to $\mathrm{F}$ ratio - 1.04:1). Their age ranged between 18 to $82 \mathrm{yrs}$. Mean age was 40.93yrs and median age was $36.5 \mathrm{yrs}$.

Among total responders $67 \%$ belongs to low income group whereas $33 \%$ were from middle income group. Being a government hospital we could not find any high income group patient.

Duration of the stay ranged from 1 to 12 days. About $45.28 \%$ responders stayed for $1-2$ days, $38.68 \%$ responders stayed in hospital for 3-5days and $16.03 \%$ responders stayed for more than 5days due to their major illness.

\section{A1. Response to process of registration, reception and admission}

As civil hospital, Palanpur, being old hospital Registration and admission protocol is in format and meticulously followed. From our study we observed that $59.70 \%$ responders from Low Income group [LIG] were satisfied with the existing procedure of registration and admission at hospital whereas $40.30 \%$ patients had complains about existing procedure. This dissatisfaction may be due to odd hour admissions keeping expectation of urgent services. In case of Middle Income group [MIG] $72.73 \%$ responders were satisfied where as $27.27 \%$ were unhappy. Overall $66.21 \%$ responders were satisfied with present procedure of registration and admission.

\section{A2. Ward and bed preparedness}

Usually the patient's cot and ward need to be prepared in anticipation of new admission at any given time. Regard to this $59.70 \%$ responders from LIG were satisfied about readiness of ward where as $40.30 \%$ were not happy. More number of MIG responders i.e. $64.64 \%$ were happy but $36.36 \%$ were having some complains.

\section{A3. Quality of food and canteen services}

Hygienic and quality food can add to early recovery of patient. Usually good hospitals take extra care for providing quality food but, our study reports high level of dissatisfaction among both LIG. It is presumed that, food provided at government hospital is of poor quality. Unhappy response expressed by $77.61 \%$ patients of LIG and $90.91 \%$ patients from MIG justifies the whispers. Similar results on food and service were also presented by Khosla JN(1969), Jain VC, Prasad BG (1969) Bhatia AK (1971). ${ }^{13-15}$

\section{A4. Rules and restraints in hospital}

Regarding maintenance of discipline in the hospital like visiting hours, restriction on number of attenders and visitors meeting patient, permission for outside food etc., both the groups were having almost equal level of dissatisfaction. $58.21 \%$ LIG were dissatisfied due to strict rule or favours; among MIG 51.52\% responders were not happy. Main dissatisfaction comes from ward boys and cleaning staff as they apply rules as per their handiness. Also a practice favouritism was reported.

Table 1: Demographic distribution of patients

\begin{tabular}{|l|c|c|}
\hline $\mathbf{n = 2 1 2}$ & No of patients & Percentage \\
\hline Male & 108 & 50.94 \\
\hline Female & 104 & 49.06 \\
\hline Age range & 18 to 82 years & \\
\hline Mean age & 40.93 years & \\
\hline Median age & 36.5 years & \\
\hline Low income group [LIG] & 142 & 66.98 \\
\hline Middle income group [MIG] & 70 & 33.01 \\
\hline Duration of stay in hospital & & \\
\hline 1 to 2 days & 96 & 45.28 \\
\hline 3 to 5 days & 82 & 38.68 \\
\hline More than 5 days & 34 & 16.03 \\
\hline
\end{tabular}

\section{A5. Cleanliness in Hospital}

Cleanliness in the hospital and surrounding is the foremost expectation of any patient visiting health care set up and it stands for the quality of hospital. ${ }^{7}$ In our study responders of both the groups equally dissatisfied with hospital environment and cleanliness. About $76.12 \%$ responders from LIG and $84.85 \%$ from MIG expressed their dissatisfaction. As present study was conducted at Government setup where lack of sincerity and dishonesty is rampant due to job security. ${ }^{11}$ 
Table 2: Response to reception restraints and cleanliness in hospital

\begin{tabular}{|l|l|c|c|c|c|}
\hline S.No & \multicolumn{1}{|c|}{ Service parameter } & \multicolumn{2}{c|}{ \% Satisfied } & \multicolumn{2}{c|}{ \% Unsatisfied } \\
\hline & & LIG & MIG & LIG & MIG \\
\hline A1 & Process of reception \& Admission & 59.70 & 72.73 & 40.30 & 27.27 \\
\hline A2 & Ward and bed preparedness & 59.70 & 64.64 & 40.30 & 36.36 \\
\hline A3 & Quality of food and canteen services & 22.39 & 9.09 & 77.61 & 90.91 \\
\hline A4 & Rules and Restraints in hospital & 41.79 & 48.48 & 58.21 & 51.52 \\
\hline A5 & Cleanliness in hospital & 23.88 & 15.15 & 76.12 & 84.85 \\
\hline
\end{tabular}

Table 3: Overall response to reception restraints and cleanliness in hospital

\begin{tabular}{|l|l|c|c|}
\hline & Service parameter & \% Satisfied & \% Unsatisfied \\
\hline A1 & Reception admission & 64 & 36 \\
\hline A2 & Ward and bed preparedness & 61 & 39 \\
\hline A3 & Quality food canteen service & 18 & 82 \\
\hline A4 & Rule restraints & 44 & 56 \\
\hline A5 & Cleanliness in hospital & 21 & 79 \\
\hline
\end{tabular}

\section{Evaluation of Soft skill: Approach and behaviour of health care staff}

Table 4: Response to approach and behaviour of health care staff

\begin{tabular}{|l|l|c|c|c|c|}
\hline S.No & \multicolumn{1}{|c|}{ Service parameter } & \multicolumn{1}{c|}{ \% Satisfied } & \multicolumn{2}{c|}{ \% Unsatisfied } \\
\hline & \multicolumn{1}{|c|}{ LIG } & MIG & LIG & MIG \\
\hline B1 & Nursing service and care at hospital & 77.61 & 78.79 & 22.39 & 21.21 \\
\hline B2 & $\begin{array}{l}\text { Cooperation and behaviour of health care provider } \\
\text { (doctors and nurse) at hospital }\end{array}$ & 76.12 & 69.70 & 23.88 & 30.30 \\
\hline B3 & Behaviour of ward and sanitary attendants & 52.24 & 48.48 & 47.76 & 51.52 \\
\hline B4 & Experience with diagnostic services & 65.67 & 69.70 & 34.33 & 30.30 \\
\hline
\end{tabular}

\section{B1. Nursing service and care at hospital: ${ }^{8}$}

Among 200 participants $78.78 \%$ expressed complete satisfaction for caring nature of nurses and their services to the patients. Only $21.22 \%$ patients were not happy with the nursing staff. This dissatisfaction may have come from chronically ill patients who seek repeated, constant and immediate attention.

\section{B2. Cooperation and behaviours of health care providers (Doctor and Nurse)}

It has been noted that fast recovery of patient depends on cordial relation of health care provider during patient stay in hospital. In our study $76.12 \%$ from LIG responders and $69.70 \%$ from MIG patients expressed contentness with the behaviour of doctors and nursing staff. Our results regarding health care provider's behaviour were similar to Sodhani PR et al (2010). ${ }^{9}$

\section{B3. Experience with diagnostic services}

Good and timely diagnostic support is a back bone of effective treatment. Paramedical staff involved in diagnostic services have important role in generating investigation reports and supporting the clinicians. In our present study we observed $69.69 \%$ responders were happy with diagnostic services and the paramedic staff.

\section{B4. Behaviour of ward and sanitary attendants}

Regarding behaviour of ward and sanitary attendant's services for patients our observations are in parallel to a

Table 6: Response to quality of medical treatment and preventive education given on patient's illness before discharge. high level of dissatisfaction among both income group responders. Overall about $52.24 \%$ responders were dissatisfied of services and behaviours of ward and sanitary attendants with patient in the hospital.

Table 5: Overall response to approach and behaviour of health care staff

\begin{tabular}{|l|c|c|}
\hline Service parameter & \%Satisfied & \% Unsatisfied \\
\hline B1. Nursing service & 78 & 22 \\
\hline $\begin{array}{l}\text { B2.Behaviour of } \\
\text { Doctors \& Nursing staff }\end{array}$ & 74 & 26 \\
\hline $\begin{array}{l}\text { B3. Behaviour of ward } \\
\text { and Sanitary attendants }\end{array}$ & 47.76 & 52.24 \\
\hline $\begin{array}{l}\text { B4. Experience with } \\
\text { diagnostic services }\end{array}$ & 67 & 33 \\
\hline
\end{tabular}




\begin{tabular}{|c|c|c|c|c|c|}
\hline S.No & Service parameter & \multicolumn{2}{c|}{ \% Satisfied } & \multicolumn{2}{c|}{ \% Unsatisfied } \\
\hline & & LIG & MIG & LIG & MIG \\
\hline C1. & Medical care and treatment at hospital & 58.21 & 66.67 & 41.79 & 33.33 \\
\hline C2. & $\begin{array}{c}\text { Preventive advice and education about illness by doctor and } \\
\text { nursing staff }\end{array}$ & 59.70 & 63.64 & 40.30 & 36.36 \\
\hline C3. & Experience of cooperation at the time of discharge & 37.31 & 36.36 & 62.69 & 63.64 \\
\hline
\end{tabular}

\section{C1. Quality of Medical care and treatment at hospital}

Overall $58.21 \%$ responders from LIG and $66.67 \%$ form MIG have expressed their satisfaction about the appropriate and rational treatment given at present general hospital by treating doctors. Overall $62 \%$ responder's satisfaction in present study disproves the belief that medical treatment at general hospital is of substandard quality. ${ }^{11}$

\section{C2. Preventive advice and education about illness by doctors and nursing staff: ${ }^{12}$}

Patient feel satisfied if they are attended and listened to their complains. They are happy when information about treatment and other detail of illness is shared and discussed with them by doctor or health care worker. In our study $63.64 \%$ MIG and $59.70 \%$ LIG responders were satisfied by the approach of doctor and other paramedics. They were also happy to receive the guidance given by doctors and nurses regarding preventive aspects of the disease they suffer. Once again total $61 \%$ responder's satisfaction disproved the common belief that doctors and nurses at government hospital are arrogant and uncaring.

\section{C3. Experience of cooperation at the time of discharge} Overall $37 \%$ responders were happy at the time of discharge. Whereas $63.64 \%$ among MIG and $62.69 \%$ from LIG responders expressed dissatisfaction towards the protocol and time taken for discharge. There are multiple reasons which caused dissatisfaction. One of the commonest is, patient wishes to reach home as early as possible after listening of discharge. They don't want any delay occurring between discharge and actual leaving the hospital. Time consumed to complete discharge procedure may be the cause of unrest.

Table 7: Overall response to quality of medical treatment and preventive education given on patient's illness before discharge

\begin{tabular}{|l|c|c|c|}
\hline S.No & Table 7 & $\begin{array}{c}\text { \% } \\
\text { Satisfied }\end{array}$ & \% Unsatisfied \\
\hline C1 & $\begin{array}{c}\text { Medical care and } \\
\text { treatment at hospital }\end{array}$ & 62 & 38 \\
\hline C2 & $\begin{array}{c}\text { Preventive education } \\
\text { about illness }\end{array}$ & 61 & 39 \\
\hline C3 & $\begin{array}{c}\text { Experience at } \\
\text { discharge }\end{array}$ & 37 & 63 \\
\hline
\end{tabular}

\section{Discussion}

There are various factors which count for patient's satisfaction from health care setup. Normally this depends on the expectations of patient from health care provider.
Patient's main expectation is getting cured from illness and re-joining their routine work without spending much time and money. ${ }^{16}$

In India, there is general perception that, health service provided at government centre is of poor quality. But with the change in legal policy of health care sector, there is drastic change in practices due to which patient's anticipations from health care provider has increased. Though there are number of factors need to be taken care to achieve patient's satisfaction, Major factors which need to be attended at all the health care centre are (1) Neat and clean hospital surrounding with friendly rules (2) Good, realistic and cordial relations adapted by health care workers (3) Time bound simple, smooth, informative and cooperative protocol for discharge.

Overall observation emerged from our present study indicates patients were satisfied and happy with the medical care and treatment provided by Civil Hospital, Palanpur. Our observations of dissatisfaction regarding behaviour of ward attendants, cleanliness of ward and hospital premises,quality of food provided in government hospitals were correlating with other study conducted in India. ${ }^{13-15}$

\section{Conclusion}

Present direct approach, exit interview study shows satisfactory response towards services and quality of health care provided by the Civil Hospital, Palanpur. Also this study identify a few shortfalls, which need to be attended for improving quality of health care services and to maintain acceptable standard of the hospital.

\section{Message}

Present direct approach exit interview method of assessment of health care services of Civil hospital from patients' responce facilitate identification of problem and help health care provider to generate ideas in solving these problems. The survey may provide feedback for quality improvement towards maintaining standard of Hospital Services.

\section{References}

1. Fereshteh Farzianpour, Raziye Byravan, Sara Amirian. Evaluation of Patient Satisfaction and Factors Affecting It: A Review of the Literature. Health 2015;07:1460-5. doi: 10.4236/health.2015.711160

2. Jingfen Shi, Yushi Jiang, Pei Hu, Yong Gong, Yuan Fng Li. A surveying study on social satisfaction to current doctor patient relationship in china. JSSM 2015;8(5):695-702.

3. Susan Abramowitz, Anne Alexis Cote RN, Elisabeth Berry. Analysing patient satisfaction: A multianalytic approach. Qual Rev Bulletin 1987;13(4)122-30.

4. Lwanga S, Lemeshow S. Sample size determination in health studies: A practical manual, Geneva, World Health Organization; 1991. 
5. Ware, JE, Snyder, MK, Wright, WR. Development and validation of scales to measure patient satisfaction with medical care services. Vol I, Part B: Results regarding scales constructed from the patients satisfaction questionnaire and measures of other health care perceptions. (NTIS Publication No PB 1976:288-329). Springfield, VA. National Technical Service.

6. Marshal GN, Hays RD. The patient satisfaction questionnaire short-form (PSQ-18). RAND Corporation, Paper P 1994:7865. Accesed at htt://www.org/health/surveys tool/psq/index.html on $12^{\text {th }}$ April 2007.

7. https://gomohealth.com/2016/7-ways-improve-patientsatisfaction-within-hospitals/

8. https://www.americansentinel.edu/about-american-sentineluniversity/newsroom/effective-nurse-communication-key-topatient-satisfaction-in-health-care-system-2

9. Sodani PR, Kumar RK, Srivastava J, Sharma L. Measuring patient satisfaction: A case study to improve quality of care at public health facilities. Indian J Community Med 2010;35:526.

10. Shubhashis Chaterjee. Attitude of supporting staff and patients satisfaction: A study in secondary health care center. IJETFT 2017;4(5):5207-12.

11. Rajinder Singh. Patient's perception towards government hospital in Haryana.VSRD TNT 2010;I(4):198-206.
12. Wanzer, M. B. Booth-Butterfield M. Gruber K. Perceptions of health care providers' communication: Relationships between patient-centered communication and satisfaction. Health Care Commun 2004;16(3):363-84.

13. Khosla JN. Citizen and Hospital Administration - A study Under Taken for Administration Reforms Commission. New Delhi: Indian Institute of Public Administration; 1969.

14. Jain VC. Prasad BG. A study hospitalised patients, Attitude towards Ward Services in the General Medical Wards of a Teaching Hospital. Ind Med Gazette 1969;9(8):3-16.

15. Bhatia AK. Patient perception of needs and problems in the hospital setup. Int J Health Educ 1971;14:145-50.

16. Houston CS, Pasanen WF. Patient's perception of hospital care. Hospitals 1972;46:70-4.

17. Reeder LG. The patient-client as a consumer: Some observations on the changing professional-client relationship. $J$ Health Soc Behav 1972;13(4):406-12.

How to cite this article: Trivedi D, Trivedi P. Evaluation of patient's satisfaction towards medical care provided at Civil Hospital, Palanpur: Assessed by direct approach exit interview method. J Manag Res Anal 2019;6(4):191-5. 